\title{
Scrolls of Descemet's membrane as unusual giant nodules on the posterior cornea: histochemical and ultrastructural findings
}

\author{
R A ALEXANDER AND A H S RAHI
}

From the Department of Pathology, Institute of Ophthalmology (University of London), 17/25 Cayton Street, London ECIV $9 A T$

SUMmARY Unusual giant nodules on the posterior surface of Descemet's membrane were observed in two out of over 400 corneae examined during routine histopathological reporting. Both of the patients, a 60-year-old man and a 26-year-old woman, had histories of corneal trauma. Neither was associated with chronic keratitis or corneal dystrophy. Light microscopy showed these nodules to be composed of material resembling Descemet's membrane. Histochemical and electron microscopical preparations identified oxytalan fibres within the outer layers of the nodules. These fibres are not a feature of the normal adult Descemet's membrane. The findings are discussed and compared with other nodular lesions of Descemet's membrane.

Descemet's membrane is an apparently homogeneous structure that is produced by the corneal endothelium. Electron microscopy, however, has shown that it consists of two distinct layers-an anterior, organised banded zone and a posterior, relatively unorganised, non-banded zone that increases in thickness with age. ${ }^{2}$ Small islands of $100 \mathrm{~nm}$ wide-spacing collagen may be observed within the periphery of the posterior layer. ${ }^{3}$ Hassal- . Henle warts are focal thickenings on the posterior surface of the peripheral Descemet's membrane; they are considered to be related to aging. ${ }^{34}$ Similar nodular thickenings in the central cornea are a feature of Fuchs's late hereditary endothelial dystrophy, while focal fusiform nodular swellings are seen in posterior polymorphous dystrophy. ${ }^{5}$ Recently Scattergood et al. ${ }^{\circ}$ described giant nodules that consisted of scrolls of Descemet's membrane in a patient with syphilitic interstitual keratitis.

If Descemet's membrane ruptures, the severed edges tend to roll posteriorly as if under a state of tension. This elastic property of Descemet's membrane is well known, though typical elastic fibres have not been demonstrated within it. ${ }^{257}$ However, Heathcote $e t$ al. ${ }^{8}$ biochemically identified desmosine and isodesmosine in bovine Descemet's membrane.

Correspondence to Mr R A Alexander.
These lysine-derived amino acids are considered to be unique to elastin. ${ }^{89}$

The mature elastic fibre is composed of two morphologically and biochemically different elements: ${ }^{11}$ a central amorphous elastin core, which may amount to $90 \%$ or more of the fibre volume, and a predominantly peripheral array of $10-16 \mathrm{~nm}$ diameter, tubular appearing, microfibrils, Oxytalan fibres $^{11}$ exist as bundles of the glycoprotein microtubules alone. They correspond to the earliest identifiable stage of elastogenesis. ${ }^{12}$ Elaunin fibres, ${ }^{13}$ the third member of the elastic fibre system, represent an intermediate stage of development between oxytalan and mature elastic, being seen as bundles of microtubules with infiltrating islands of amorphous material. ${ }^{14}$ The histochemical properties of the elastic fibre family are outlined in Table 1.

As with mature elastic, neither oxytalan nor elaunin fibres are demonstrable in the normal adult human cornea.' Oxytalan fibres though, have been observed as a transient feature of the young Descemet's membrane of $\operatorname{man}^{7}$ and cat. ${ }^{15}$ They have also been identified within the retrocorneal fibrous tissue layer produced by endothelial cells in examples of Fuchs's endothelial dystrophy, ${ }^{16}{ }^{17}$ congenital endothelial dystrophy, ${ }^{16}$ posterior polymorphous dystrophy, ${ }^{16}$ and advanced keratoconus. ${ }^{18}$ The production of oxytalan fibres in later life, as seen in these 
Table 1 Summary of the histochemical properties of oxytalan, elaunin, and mature elastic fibres

\begin{tabular}{llll}
\hline & \multicolumn{3}{l}{ Fibre type } \\
\cline { 2 - 4 } Methods & $\begin{array}{l}\text { Mature } \\
\text { elastic }\end{array}$ & Elaunin & Oxytalan \\
\hline Verhoeff iron haematoxylin & + & - & - \\
Ox-Verhoeff iron haematoxylin & + & - & - \\
Gomori aldehyde fuchsin & + & + & - \\
Ox-aldehyde fuchsin & + & + & + \\
Elastase-Verhoeff iron haematoxylin & A & - & - \\
Elastase-Aldehyde fuchsin & A & A & - \\
Elastase-ox-aldehyde fuchsin & A & S* $^{*}$ & S \\
Ox-elastase-aldehyde fuchsin & A & A & A \\
\hline
\end{tabular}

$O x=$ section oxidation in $10 \%$ Caroat. $-=$ unstained. $+=$ stained. $\mathrm{S}=$ stained (little or no change). $\mathrm{A}=$ abolished staining.

*Staining of amorphous core abolished by elastase but peripheral microfibrils induced to stain by oxidation.

corneal diseases, may be the result of derepression of fetal genes.

In a review of more than 100 corneal discs and over 300 eyes which were removed for a variety of reasons we found two examples of giant nodules on the posterior surface of Descemet's membrane which were morphologically similar to those of Scattergood et al. ${ }^{6}$

The present communication describes the distribution of elastic related fibres in our two cases with giant nodules of Descemet's membrane. The findings are compared with those of other nodular lesions of Descemet's.

\section{Materials and methods}

Specimen 1 was an eye enucleated for malignant melanoma of the choroid. The patient was a 60 -yearold man who had a history of perforating injury to the cornea during childhood.

Specimen 2 was an eye from a 26-year-old woman. It was painful and blind as a consequence of longstanding raised intraocular pressure (congenital glaucoma). There was evidence of previous iridectomy.

Neither of the patients had clinical evidence of either corneal dystrophy or chronic keratitis.

\section{LIGHT MICROSCOPY}

Both eyes were fixed in $10 \%$ formal saline, processed into paraffin wax, and sectioned at a thickness of $6 \mu \mathrm{m}$.

Sections were dewaxed, hydrated, and stained by the following methods:

Mayer's haematoxylin and eosin for routine examination.

Verhoeff's iron haematoxylin for mature elastic fibres.
Gomori's aldehyde fuchsin for elaunin and mature elastic fibres.

Aldehyde fuchsin following oxidation in $10 \%$ caroat for $60 \mathrm{~min}$ or peracetic acid for $30 \mathrm{~min}^{19}$ to demonstrate oxytalan fibres in addition to elaunin and elastic. Oxytalan fibres are identified histochemically by the fact that they stain with aldehyde fuchsin only when tissue sections have been previously exposed to a strong oxidising agent. ${ }^{11} 1219$ (Caroat, active component potassium peroxymonosulphate, was obtained from Degussa Ltd, Cheadle Hulme, Cheshire.)

Gomori's silver impregnation method for reticular fibres.

Gomori's one step trichrome for collagen.

McManus periodic acid Schiff sequence.

\section{TRANSMISSION ELECTRON MICROSCOPY}

One of the two nodules on the posterior cornea of case 2 was excised from the wax block, dewaxed, rehydrated, and postfixed in $1 \%$ osmium tetroxide in Palade buffer at $\mathrm{pH} 7 \cdot 4$. The specimen was subsequently processed and embedded in Araldite. Thin sections were stained by alcoholic uranyl acetate and lead citrate and studied under a Joel $100 \mathrm{C}$ electron microscope.

\section{Results}

CASE 1

The most significant abnormality was the presence of a giant dome-shaped nodule on the posterior surface of the central cornea. It measured approximately $0.4 \times 0.19 \mathrm{~mm}$ in cross section. The central cornea also showed signs of previous perforating injury, such as deficiencies of Bowman's zone, disorganisation of the stromal lamallae, and fragmentation of Descemet's membrane. The basal or stromal aspect of the nodule was seen to be composed of fibrous scar tissue which had penetrated through the break in Descemet's membrane. The 'new Descemet's membrane' which covered the scar tissue and constituted the giant nodule was greatly thickened, in contrast to the old Descemet's membrane with which it was continuous. A few small guttate excrescences or warts were seen immediately to one side of the nodule.

Many oxytalan fibres were identified within the posterior or outer layers of the thickened Descemet's membrane/nodule. These fibres outlined several buried and hence unsuspected warts within the nodule in addition to those previously observed. Where oxytalan fibres penetrated between individual warts they imparted a fern leaf pattern to the nodule. Oxytalan fibres as well as presenting as distinct fibres were also seen as dots. This appearance was interpre- 
ted as being fibres cut in cross section and hence encircling the warts in the nodule. Oxytalan was not observed in the more anterior or inner layers of the nodule. A relatively small number of oxytalan fibres were seen immediately beneath the endothelium over most of Descemet's membrane. A few very fine oxytalan fibres were present beneath the basal cells of the central corneal epithelium and superficial stroma in the region of the old perforating wound.

Elaunin and mature elastic fibres were not identified within any part of the cornea. The severed ends of the original Descemet's membrane observed within the nodule were, however, weakly stained by the Verhoeff elastic method, but not by aldehyde fuchsin - with or without preliminary oxidation.

Argyrophillic reticular fibres were shown by the
Gomori silver method to give the nodule and Descemet's membrane a multilayered appearance. Reticular fibres were present in the anterior layers of the nodule as well as within the oxytalan-containing posterior layers. As with oxytalan, some reticular fibres were considered to encircle the guttate excrescences.

CASE 2

Two large nodules were seen to project from the posterior surface of the cornea. The nodules appeared to be formed by the infolding of a greatly thickened Descemet's membrane. The centre of both nodules contained severed and spiraled fragments of the original Descemet's membrane. The two large nodules measured $0.14 \times 0.2 \mathrm{~mm}$ and $0.2 \times 0.1 \mathrm{~mm}$ in
Fig. 1 One of the giant nodules from case 2. The severed and coiled Descement's membrane is seen within the nodule. Fine oxytalan fibres (arrows) are only seen within the more posterior or outer regions of the nodule. These fibres outline unsuspected guttate excresences $(\mathrm{g})$. (Oxidation-aldehyde fuchsin, $\times 544)$. Inset. If the oxidation step of the technique is omitted, oxytalan fibres fail to stain. Elaunin and elastic fibres are not present. (Aldehyde fuchsin, $\times 136$ ).

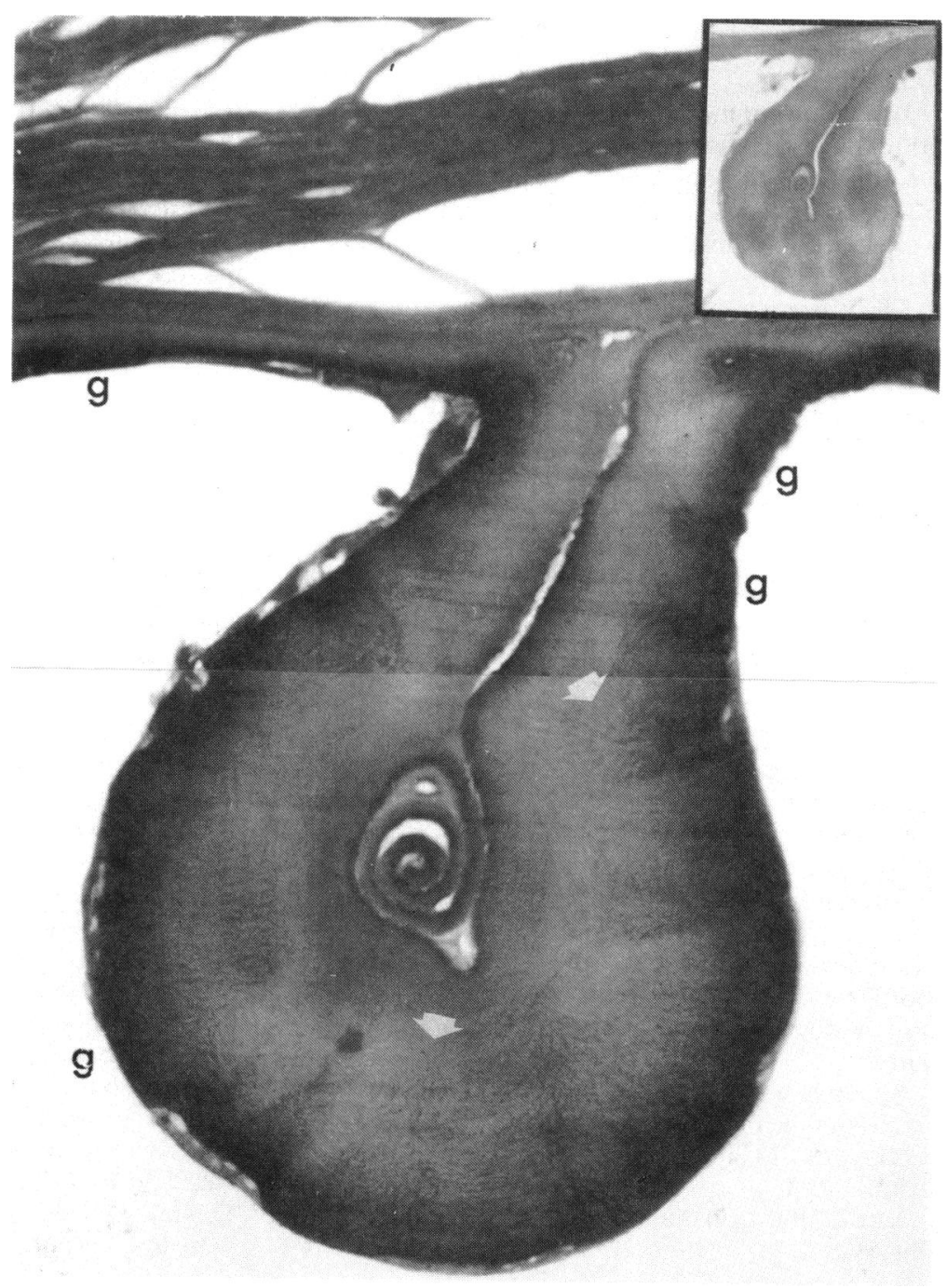


cross section. The posterior surface of Descemet's membrane on either side of the nodules had occasional small focal swellings or guttate excrescences.

A great many fine oxytalan fibres were identified within the more posterior-outer layers of the thickened Descemet's membrane which constituted the giant nodules (Figs. 1 and 2). The oxytalan fibre staining outlined and so revealed numerous buried individual and multiple unsuspected guttate warts within the nodules. The excrescences of Descemet's membrane proper were also outlined by oxytalan fibres. Oxytalan fibres were not present within the anterior-inner layers of the nodules. A small number of very fine fibres were demonstrated beneath the corneal epithelium and within the superficial stroma.

Elaunin and elastic fibres were not seen within the cornea. However, as with case 1 , the coiled, severed ends of Descemet's membrane within the nodules were weakly stained by the Verhoeff technique but not by the aldehyde fuchsin methods.

Argyrophilic reticular fibre staining gave a multilaminar appearance to both the normal Descemet's membrane and the nodules.

Transmission electron microscopy of the giant nodule shown in Fig. 1 revealed four morphologically different layers or zones. The first two layers, at the centre of the nodule, were formed from the anterior banded and posterior non-banded zones of the coiled, severed ends of Descemet's membrane. The third layer, which formed the bulk of the nodule and the buried excrescences, resembled the banded zone of Descemet's membrane proper. In addition it contained fine fibrils and basement membrane or ground substance material. This latter material cor- responds to the reticular fibres seen by light microscopy. The fourth and outermost layer (Fig. 3) appeared less compact than the others and was of variable thickness. It was seen to fill the spaces between and around the individual excrescences to give a smooth outline to the nodule. This loose fibrous layer consisted of collagen fibrils, reticular fibres, and bundles of $12 \mathrm{~nm}$ diameter microtubules characteristic of oxytalan. Oxytalan fibres were not observed in the other zones of the nodule. The absence of elaunin and elastic fibres was confirmed.

\section{Discussion}

Oxytalan fibres have been identified in several conditions of the cornea associated with thickening of Descemet's membrane. ${ }^{16-18}$ In congenital hereditary endothelial dystrophy Descemet's membrane is uniformly thickened by the production of a fibrillar collagenous layer posterior to the membrane proper. ${ }^{20}$ In addition to possessing collagen and basement membrane material the layer shows a great many randomly orientated oxytalan fibres. ${ }^{16}$ Likewise Descemet's membrane of the central cornea may be thickened in cases of advanced keratoconus. The retrocorneal fibrous membrane shows many roughly parallel oxytalan fibres lying at approximately $80^{\circ}$ to the posterior surface of Descemet's membrane.$^{18}$ In posterior polymorphous dystrophy the abnormal posterior fibrous layers form discrete fusiform nodular swellings. ${ }^{20}$ Oxytalan fibres are present within this tissue and are seen to outline the posterior surfaces of the swellings. ${ }^{16}$ They are not apparent within the nodules or elsewhere in

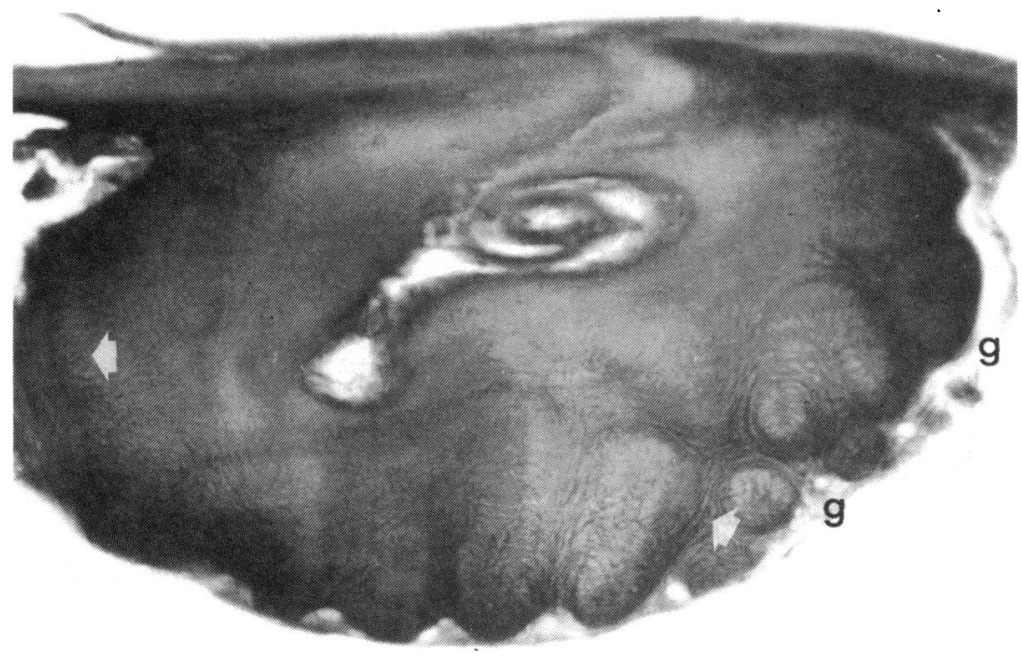

Fig. 2 The other giant nodule from case 2. Oxytalan fibres (arrows) reveal multiple guttae (g) within the outer aspects of the nodule. (Oxidation-aldehyde fuchsin, $\times 436$ ). 
Descemet's membrane.${ }^{16}$ Certain disease states, most notably Fuchs's endothelial dystrophy, show many dome or mushroom shaped guttate excrescences along the posterior surface of a thickened Descemet's membrane. ${ }^{521}$ Iwamoto and DeVoe ${ }^{21}$ showed that the thickened membrane consisted of up to five different collagenous layers: the anterior banded and posterior non-banded zones of Descemet's membrane; a posterior banded layer which also formed the warts; a border region containing 'thin fibrils' in addition to wide-spacing collagen, collagen fibrils, and basement membrane material; and a fibrillar layer similar to the border layer but which did not show wide-spacing collagen. Oxytalan fibres are

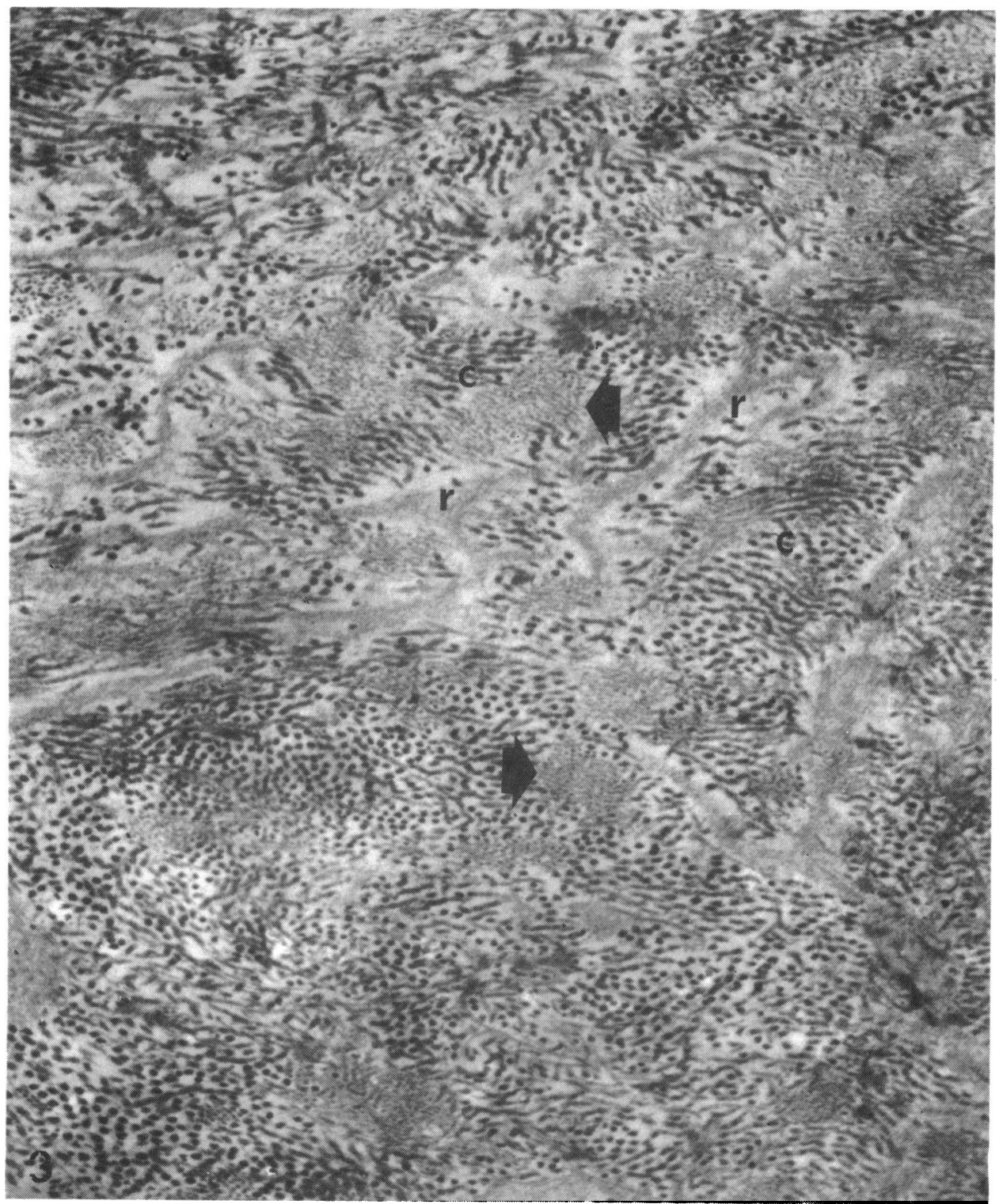

Fig. 3 Electron micrograph showing part of the loose fibrous outermost layer of the nodule shown in Fig. 1. Numerous oxytalan fibres (arrows), composed of aligned $12 \mathrm{~nm}$ diameter fibrils are seen. Some of these 'islands' of oxytalan probably represent successive sightings of the same long fibre. Small diameter collagen fibrils (c) are mainly shown as cross-sectioned 'dots'. Basement membrane or reticular fibres (r) are also present. (Uranyl acetate-lead citrate, $\times 37500$ ). 
present in large amounts within both the border and fibrillar layers of the retrocorneal collagenous membrane. ${ }^{16}{ }^{17}$ Oxytalan thus outlined the warts but was not present within them. Significant numbers of oxytalan fibres lay parallel to the surface of Descemet's membrane, where they encircled individual excrescences. ${ }^{17}$ Reticular fibres (basement membrane material) were present within the posterior banded layer as well as the two layers containing oxytalan fibres. ${ }^{17}$ Similar warts commonly occur in small numbers on the peripheral cornea (Hassal-Henle bodies) or over the central cornea as a part of normal endothelial aging. ${ }^{34}$ Oxytalan fibres are present in small numbers over the posterior surface only of most Hassal-Henle warts. ${ }^{7}$

If the giant nodules of our study could be unfolded and flattened, then on ultrastructural morphology and oxytalan fibre staining they would most closely resemble the posterior structures of Fuchs's endothelial dystrophy. However, case 1, a 60-year-old, did not have guttate excrescences on the central cornea unassociated with the nodule. Case 2, a 26year-old, did have a small number of warts scattered over Descemet's membrane and therefore could conceivably be an early example of Fuchs's dystrophy. It is interesting that the nodules of both cases should contain increased numbers of excrescences as compared with the rest of Descemet's membrane.

To the best of our knowledge this report is the first detailed documentation of the fibrous tissue nature of giant nodules of Descemet's membrane. There was no sex or age predilection, but both patients had a history of corneal injury resulting in a transient lowering of intraocular pressure. Since wrinkles and folds in Descemet's membrane are constant features of ocular hypotony,,$^{22}{ }^{23}$ it is conceivable that such folds may have developed in the cases under discussion. These folds may have been maintained for a considerable time, resulting in altered endothelial activity and overproduction of connective tissues, thus forming the giant nodules.

Multilaminar thickenings of Descemet's membrane, secondary focal guttae, and retrocorneal hyaline ridges have been observed in patients with interstitial keratitis,$^{20}$ and scrolls of Descemet's membrane forming giant nodules have been reported in one case. ${ }^{6}$ Neither of our cases had clinical or histological evidence of interstitial keratitis, and therefore such giant nodules of Descemet's membrane do not appear to be pathognomonic of interstitial keratitis.

\section{References}

1 Fine BS, Yanoff M. Ocular histology. A text and atlas. New York: Harper and Row, 1972.

2 Tripathi RC. Fine structure of mesodermal tissues of the human eye. Trans Ophthalmol Soc UK 1974; 94: 663-95.

3 Feency ML, Garron LK. Descemet's membranc in the human peripheral cornea. In: Smelser GK, cd. The structure of the eye. New York: Academic Press, 1961: 367-80.

4 Lorenzetti DW, Uotila MH, Parikh RN, Kaufman HE. Central cornea guttata. Incidence in the general population. $A m \mathrm{~J}$ Ophthalmol 1967; 64: 1155-967.

5 Klintworth GK. The cornea-structure and macromolecules in health and disease. A review. Am J Pathol 1977; 89: 718-808.

6 Scattergood KD, Green WR, Hirst LW. Scrolls of Descemet's membrane in healed syphilitic interstitial keratitis. Ophthalmology (Rochester) 1983; 90: 1518-23.

7 Alexander RA, Garner A. Elastic and precursor fibres in the normal human eye. Exp Eye Res 1983; 36: 305-15.

8 Heathcote JG, Eyre DR, Cross J. Mature bovine Descemet's membrane contains desmosine and isodesmosine. Biochem Biophys Res Comm 1982; 108: 1588-94.

9 Ross R, Bornstein P. The elastic fiber. I. The separation and partial characterization of its macromolecular components. J Cell Biol 1969; 40: 366-81.

10 Cleary EG, Fanning JC, Prosser I. Possible roles of microfibrils in elastogenesis. Connect Tissue Res 1981; 8: 161-6.

11 Fullmer HM, Lillic RD. The oxytalan fibre: a previously undescribed connective tissue fibre. J Histochem Cytochem 1958; 6: $425-30$.

12 Fullmer HM, Sheetz JH, Nakates AJ. Oxytalan connective tissue fibres: a review. J Oral Pathol 1974; 3: 291-316.

13 Gawlik Z. Morphological and morphochemical properties of the elastic system of the motor organ of man. Folia Histochem Cytochem (Krakow) 1965; 3: 233-51.

14 Cotta-Pereira G, Guerra-Rodrigo F, Bittencourt-Sampaio S. Oxytalan, elaunin and elastic fibres in the human skin $J$ Invest Dermatol 1976; 66: 143-8.

15 Carrington SD, Alexander RA, Gricrson I. Elastic and related fibres in the normal cornea and limbus of the domestic cat. $J$ Anat 1984; 139: 319-32.

16 Garner A, Alexander RA. Pre-elastic (oxytalan) fibres in corneal pathology. In: The cornea in health and disease. VIth Congress European Society of Ophthalmology, Brighton 1980. London: Royal Society of Medicine-Academic Press, $1981 ; 213-6$.

17 Alexander RA, Grierson I, Garner A. Oxytalan fibres in Fuchs' endothelial dystrophy. Arch Ophthalmol 1981; 99: 1622-7.

18 Alexander RA. Elastic and related fibres in the human cornea. M.Phil thesis. University of London, 1981.

19 Alexander RA, Clayton DC, Howes RC, Garner A. The effect of oxidation upon the demonstration of corneal oxytalan fibresa light and electron microscopical study. Med Lab Sci 1981; 38: 91-101.

20 Waring GO, Rodrigues MM, Laibson PR. Corneal dystrophies II. Endothelial dystrophies. Surv Ophthalmol 1978; 23: 147-68.

21 Iwamoto T, DeVoe AG. Electron microscopic studies on Fuchs' combined dystrophy I. Posterior portion of the cornea. Invest Ophthalmol Vis Sci 1971; 10: 9-28.

22 Moreau, Cornibert, Mugneret. Hypotonie grave par dénutrition. Bull Soc Ophtalmol Fr 1963: 243-6.

23 Murthy G, Adrianwala SD. Bilateral Descemet's wrinkles in acute gastro-enteritis. Indian J Ophthalmol 1984; 34: 229-30.

Accepted for publication 30 May 1985. 